\title{
Treatment of Crimean-Congo Haemorrhagic Fever by Favipiravir in a Patient with Novel Coronavirus Co-Infection
}

\author{
Ahmet Cumhur Dülger ${ }^{1}$, Mustafa Yakarişik² ${ }^{2}$ Yusuf Emre Uzun ${ }^{3}$, Ahmet Melih Şahin ${ }^{4}$ \\ ${ }^{1}$ Department of Gastroenterology, Giresun State Hospital Affiliated to Giresun University,Giresun, Turkey \\ ${ }^{2}$ Department of Internal Medicine, Giresun State Hospital Affiliated to Giresun University,Giresun, Turkey \\ ${ }^{3}$ Department of Internal Medicine, Dereli State Hospital, Giresun, Turkey \\ ${ }^{4}$ Department of Infectious Disease, Giresun State Hospital Affiliated to Giresun University, Giresun, Turkey
}

\section{Doi: 10.12890/2020_002042 - European Journal of Case Reports in Internal Medicine - ๑ EFIM 2020}

Received: 05/10/2020

Accepted: $14 / 10 / 2020$

Published: $17 / 12 / 2020$

How to cite this article: Dülger AC, Yakarişik M, Uzun YE, Şahin AM. Treatment of Crimean-Congo haemorrhagic fever by favipiravir in a patient with novel coronavirus co-infection. EJCRIM 2020;7: doi:10.12890/2020_002042.

Conflicts of Interests: The Authors declare that there are no competing interests.

This article is licensed under a Commons Attribution Non-Commercial 4.0 License

\section{ABSTRACT}

Introduction: Crimean-Congo haemorrhagic fever (CCHF) is a lethal zoonotic disease caused by an RNA virus that is a member of the Nairovirus genus in the Bunyaviridae family from the arbovirus group. CCHF is transmitted by Hyalomma ticks through direct contact with the blood and other bodily fluids of patients or infected animals.

Case description: A 65-year-old man was admitted to the emergency unit with dry cough, myalgia and fever. He was treated with favipiravir. He had disseminated intravascular coagulopathy with thrombocytopenia in the setting of COVID-19 infection. He tested positive for both COVID-19 and CCHF. By the end of the fifth day of treatment, his laboratory parameters and clinical symptoms had normalized.

Conclusion: Favipiravir is currently on the market for treating COVID-19 infection worldwide. It has also been used to treat CCHF in laboratory animals. To the best of our knowledge this is the first report of CCHF successfully treated with favipiravir, which could be a key drug for treating human CCHF.

\section{LEARNING POINTS}

- Clinicians should be alert for concomitant viral infections such as Crimean-Congo haemorrhagic fever, which share similar clinical and laboratory findings to COVID-19.

- The effectiveness of favipiravir for viral infections other than influenza and COVID-19, such as Crimean-Congo haemorrhagic fever, should be elucidated.

\section{KEYWORDS}

Crimean-Congo haemorrhagic fever, COVID-19, favipiravir

\section{INTRODUCTION}

Crimean-Congo haemorrhagic fever (CCHF) is a lethal zoonotic disease caused by an RNA virus that is a member of the Nairovirus genus in the Bunyaviridae family from the arbovirus group. The virus contains negative-stranded, enveloped RNA. CCHF is transmitted by Hyalomma ticks through direct contact with the blood and other bodily fluids of patients or infected animals. The CCHF incubation period is $2-14$ days and correlates with the type of transmission and viral load (2-7 days following a tick bite or 10-14 days after blood transfusion). The spectrum of clinical manifestations ranges from subclinical illness to acute infection with haemorrhage (petechiae, ecchymoses, epistaxis, pulmonary haemorrhage, intra-abdominal bleeding, haematuria, melena) and multiorgan failure ${ }^{[1]}$. 
CCHF is widely distributed from the Black Sea to southern Africa. It has a high case fatality rate of approximately $30 \%$, and is predominantly treated with supportive care; the efficacy of ribavirin is controversial ${ }^{[2]}$. Novel coronavirus (COVID-19) infection has developed into a global pandemic, is associated with increased rates of mortality and has well-established risk factors including diabetes mellitus, ischaemic heart disease and cancer ${ }^{[3]}$.

Favipiravir, a pyrazine analog, is a potent and selective RNA-dependent RNA polymerase inhibitor of RNA viruses ${ }^{[4]}$. It has potent antiviral activity against influenza virus, and also has antiviral activity in vitro and in animal models against hantaviruses, alphaviruses, flaviviruses and norovirus ${ }^{[5]}$.

COVID-19 infection is characterized by fever, shortness of breath, higher D-dimer levels, lymphopenia, and pulmonary infiltrates including ground-glass opacities on CT of the chest ${ }^{[6]}$. To the best of our knowledge, there have been no previous reports of CCHF with concomitant COVID-19 treated with favipiravir. We describe a patient with acute CCHF infection who developed disseminated intravascular coagulopathy with thrombocytopenia in the setting of concomitant COVID-19 infection.

\section{CASE DESCRIPTION}

The patient was a 65-year-old male farmer. He presented to the emergency room with dry cough, myalgia and fever. His medical history included hypertension and benign prostate hyperplasia. His regular medications were perindopril/indapamide/amlodipine and silodosin. His physical examination results were as follows: pulse 84 beats per minute, blood pressure $120 / 80 \mathrm{mmHg}$, body temperature $36.7^{\circ} \mathrm{C}$, and oxygen saturation $95 \%$ on ambient air. His complete blood count revealed: WBC $1.32 \times 10^{9} / \mathrm{l}$, Neu 0.63×10\%/l, Lymp 0.55×10\%/l, Hgb $11.6 \mathrm{~g} /$ dl, and PIt 50×10\%/. Laboratory studies showed: CRP 5.51 mg/l, D-dimer 7909 ng/ml, ferritin $86.053 \mu \mathrm{g} / \mathrm{l}, \mathrm{CK} 726 \mathrm{U} / \mathrm{I}, \mathrm{LDH} 1357 \mathrm{U} / \mathrm{l}$, ALT 125 U/I, AST 349 U/I, PT 10.5 s, INR 0.9, and fibrinogen 242 mg/dl.

There were questionable areas of thrombocytopenia and because the clinical course suggested another viral infection, blood samples were taken to test for COVID-19, CCHF and hantavirus. Computed tomography (CT) of the chest revealed multiple bilateral peripheral groundglass opacities consistent with acute viral infection.

RT-PCR amplification of SARS-CoV-2 virus nucleic acid from a nasopharyngeal swab was positive. Molecular analysis of blood by RT-PCR showed that the patient was also positive for CCHF disease. The remainder of the serological work-up was negative. The patient was treated with favipiravir $1600 \mathrm{mg}$ twice on the first day, and $600 \mathrm{mg}$ twice a day for the following 4 days. After 5 days of treatment, the patient had responded well to treatment with favipiravir. Blood analysis showed: WBC $3.65 \times 10^{9} / \mathrm{l}, \mathrm{Neu} 2.46 \times 10^{9} / \mathrm{l}, \mathrm{Lymp} 0.86 \times 10^{9} / \mathrm{I}$, Hgb $11.5 \mathrm{~g} / \mathrm{dl}$,

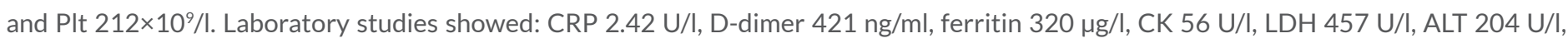
AST 133 U/I, PT 10.2 s, INR 0.88, and fibrinogen 320 mg/dl. His symptoms of fever and shortness of breath also improved in line with a decrease in serum CRP levels, while the last two consecutive nasopharyngeal swabs were negative. The patient was finally discharged with a recommendation for self-isolation and tele-medicine.

\section{DISCUSSION}

Here we have described a CCHF patient with COVID-19 co-infection. He was treated with favipiravir because he had several positive COVID-19 PCR tests. COVID-19 infection is a novel viral disease mainly of the pulmonary system that is characterized by fever, cough, myalgia, shortness of breath, ground-glass opacities and multiple infiltrates on CT of the chest, lymphopenia, hyperferritinaemia, and higher CRP and D-dimer levels ${ }^{[3]}$. Our clinical, laboratory and radiological findings were consistent with COVID-19 infection as presented. In addition, it is very important to accurately identify COVID-19 infection using proper medical tests to prevent misdiagnosis amid this pandemic. Thus, we performed PCR testing together with other laboratory investigations as well as CT of the chest during the initial examination as described above.

Anaemia, thrombocytopenia, leukopenia, hyperbilirubinemia, elevated transaminases, increased levels of both blood urea nitrogen and serum creatinine, higher levels of creatine phosphokinase and prolongation of the international normalized ratio, decreased fibrinogen levels and increased fibrin-degradation products are seen in laboratory tests in CCHF ${ }^{[7]}$. All laboratory findings of the presented case were compatible with CCHF. Diagnostic tools include detection of CCHF virus (CCHFV) RNA by RT-PCR and specific immunoglobulin (Ig)M and IgG by enzyme-linked immunosorbent assay ${ }^{[8]}$.

In our patient, RT-PCR tests were positive for CCHF. Favipiravir (6-fluoro-3-hydroxy-2-pyrazinecarboxamide) is a pseudo purine analogue which has been recently tested and found effective for the treatment of COVID-19 infection ${ }^{[9]}$. Although there is still lack of vaccines and recommended antiviral treatment for patients with CCHF, Oestereich et al. reported that mice infected with the CCHF virus were successfully treated with favipiravir with all treated animals surviving with no viral load ${ }^{[10]}$. Similarly, another animal study also revealed that favipiravir had clinical benefits even if administered late in the course of CCHF ${ }^{[11]}$. However, it is important to note that these in vivo studies were conducted in animals. Thus, the risks and potential benefits of favipiravir are unknown in case of CCHF infection in human. 
Our patient was living in a region endemic for CCHF, and was also infected with SARS-CoV-2. Thus, we also suspected CCHF because of thrombocytopenia, leukopenia, increasing INR and higher D-dimer level. In our case, we used favipiravir on the first hospitalization day as a standard therapy to treat the COVID-19 infection and indirectly CCHF. As expected, the patient's laboratory parameters normalized, he clinically recovered and was discharged home with close tele-medicine observation.

To our knowledge, this is the first report of CCHF with COVID 19 co-infection which was successfully treated with favipiravir. Further research is needed to elucidate the effectiveness of favipiravir in viral infections, especially CCHF.

\section{REFERENCES}

1. Shayan S, Bokaean M, Shahrivar MR, Chinikar S. Crimean-Congo hemorrhagic fever. Lab Med 2015;46(3):180-189

2. Messina JP, Pigott DM, Golding N, Duda KA, Brownstein JS, Weiss DJ, et al. The global distribution of Crimean-Congo hemorrhagic fever. Trans R Soc Trop Med Hyg 2015;109(8):503-513.

3. Taisheng L. Diagnosis and clinical management of severe acute respiratory syndrome Coronavirus 2 (SARS-CoV-2) infection: an operational recommendation of Peking Union Medical College Hospital (V2.0). Emerg Microbes Infect 2020;9(1):582-585.

4. Furuta Y, Komeno T, Nakamura T. Favipiravir (T-705), a broad spectrum inhibitor of viral RNA polymerase. Proc Jpn Acad Ser B Phys Biol Sci $2017 ; 93$ (7):449-463.

5. Delang L, Abdelnabi R, Neyts J. Favipiravir as a potential countermeasure against neglected and emerging RNA viruses. Antivir Res 2018;153:85-94.

6. Acter T, Uddin N, Das J, Akhter A, Choudhury TR, Kim S. Evolution of severe acute respiratory syndrome coronavirus 2 (SARS-CoV-2) as coronavirus disease 2019 (COVID-19) pandemic: a global health emergency. Sci Total Environ 2020;730:138996.

7. Hawman DW, Feldmann H. Recent advances in understanding Crimean-Congo hemorrhagic fever virus. F1000Res 2018;7:F1000 Faculty Rev-1715.

8. Sas MA, Vina-Rodriguez A, Mertens M, Eiden M, Emmerich P, Chaintoutis SC, et al. A one-step multiplex real-time RT-PCR for the universal detection of all currently known CCHFV genotypes. J Virol Methods 2018;255:38-43.

9. Zhang J, Xie B, Hashimoto K. Current status of potential therapeutic candidates for the COVID-19 crisis. Brain Behav Immun 2020;87:59-73.

10. Oestereich L, Rieger T, Neumann M, Bernreuther C, Lehmann M, Krasemann S, et al. Evaluation of antiviral efficacy of ribavirin, arbidol, and T-705 (favipiravir) in a mouse model for Crimean-Congo hemorrhagic fever. PLoS NegI Trop Dis 2014;8(5):e2804.

11. Hawman DW, Haddock E, Meade-White K, Williamson B, Hanley PW, Rosenke K, et al. Favipiravir (T-705) but not ribavirin is effective against two distinct strains of CrimeanCongo hemorrhagic fever virus in mice. Antivir Res 2018;157:18-26. 